\title{
Aggregation of fuzzy preferences: some rules of the mean ${ }^{\dagger}$
}

\author{
José Luis García-Lapresta \& Bonifacio Llamazares
}

Universidad de Valladolid (Spain)

Dep. de Economía Aplicada (Matemáticas)

Facultad de Ciencias Económicas y Empresariales

Running title: Aggregation of fuzzy preferences.

Corresponding author:

José Luis García Lapresta.

Dep. de Economía Aplicada (Matemáticas).

Facultad de Ciencias Económicas y Empresariales.

Avda. Valle de Esgueva, 6. 47011 Valladolid, Spain.

Phone: $34+983+423330$.

Fax: $34+983+423299$.

E-mail: lapresta@cpd.uva.es

\footnotetext{
${ }^{\dagger}$ The finantial support of the Junta de Castilla y León (Consejería de Educación y Cultura, Proyecto VA09/98) is gratefully acknowledged. The authors are indebted to Maurice Salles and two anonymous referees for their valuable comments and helpful suggestions.
} 


\begin{abstract}
This paper studies by means of reciprocal fuzzy binary relations the aggregation of preferences when individuals show their preferences gradually. We have characterized neutral aggregation rules through functions from powers of the unit interval in the unit interval. Furthermore, we have determined the neutral aggregation rules that are decomposable and anonymous. In this class of rules, the collective intensity of preference is the arithmetic mean of the values assigned by a function to the individual intensities of preference. We have also considered the neutral aggregation rules based on quasiarithmetic means. We have established that this class of rules generalizes the simple majority, when individuals have ordinary preferences and collective preferences are reciprocal.
\end{abstract}

\title{
1 Introduction
}

A classical problem of Social Choice Theory consists in finding collective preferences from individual preferences, so that collective decisions can be taken, observing some ethical principles. In most cases it has been supposed that individuals have exact preferences. However, individuals generally prefer some alternatives to others with different levels of intensity. Fuzzy binary relations reflect this information, evaluating the levels of preference intensity between 0 and 1 . Reciprocity assures that the sum of preference intensities among alternatives is always 1. Taking individual levels of intensity of preference into account, collective decisions can more faithfully observe individuals' desires than the conventional case, where preferences are not gradual.

Following Bellman-Zadeh work [4], contributions of fuzzy set theory to group decision making have been numerous. See, for example, Blin-Whinston [8], Blin [9], Nurmi [31], Bezdek-SpillmanSpillman [5] and [6], Dubois-Prade [17], Barrett-Pattanaik-Salles [2], Yager [36] and [37], DuboisKoning [16], Billot [7], Barrett-Pattanaik-Salles [3], and Fodor-Roubens [22, chapter 5], among others.

In this paper we consider aggregation rules which assign an aggregate fuzzy binary relation to each profile of reciprocal fuzzy binary relations. The aggregate fuzzy binary relation does not necessarily have to be reciprocal, but it is desirable for it to be so. When this relation is reciprocal, collective decisions can be taken with different levels of qualification, depending upon the ordinary preference relation that is considered based on the aggregate fuzzy relation. In our paper we characterize neutral aggregation rules through functions from powers of $[0,1]$ in $[0,1]$. In addition, we define a class of neutral aggregation rules based on arithmetic means associated with functions of $[0,1]$. These rules are characterized through two properties: decomposability and anonymity. Furthermore, we characterize rules which satisfy additional properties. 
We also consider neutral aggregation rules defined by quasiarithmetic means associated with order automorphisms of $[0,1]$. Based on a classical result of Kolmogoroff [26] and Nagumo [29], five properties characterize this class of rules: anonymity, unanimity, continuity, strict monotonicity and associativity. Ovchinnikov [34, Th. 5.1] characterizes the quasiarithmetic aggregation rules, when individuals have ordinary preferences, by means of some properties of fuzzy aggregation; the restrictions of these properties on the ordinary case are similar to those given by May [28] for simple majority. We characterize reciprocal quasiarithmetic aggregation rules and justify the reason why they generalize the simple majority rule, when individuals have ordinary preferences. Special majorities have been studied by Fishburn [21, chapter 6] and Ferejohn-Grether [20], among others.

Algebraic and topological means have been studied by Aumann [1], Eckmann [18], EckmannGanea-Hilton [19]. Applications to social choice theory can be found in Chichilnisky-Heal [13], Candeal-Induráin-Uriarte [11], Candeal-Induráin [12] and Horvath [24], [25], among others. Moreover, in Bullen-Mitrinović-Vasić [10] there is an exhaustive study on means.

The organization of the paper is as follows. Firstly we introduce our notation and the basic concepts employed. Section 2 characterizes neutral aggregation rules by means of auxiliary functions of powers of $[0,1]$ in $[0,1]$ (Theorem 1 ). Moreover, we present neutral aggregation rules that satisfy additional properties, such as anonymity, unanimity, reciprocity, monotonicity, strict monotonicity, continuity, uniformity, additivity, decomposability and associativity, through the auxiliary functions. We also show some relations among the properties mentioned. Section 3 is devoted to characterizing the neutral aggregation rules that are decomposable and anonymous (Theorem 2). In this class of rules, we characterize uniformity (Corollary 2), unanimity (Corollary 3), additivity (Corollary 4), monotonicity and strict monotonicity (Corollary 5), reciprocity (Corollary 6) and continuity (Corollary 7). Section 4 is concerned with neutral aggregation rules where the auxiliary functions are defined by means of quasiarithmetic means associated with order automorphisms of $[0,1]$. We characterize reciprocity in the aggregation rules of quasiarithmetic means (Proposition 1). Finally, Section 5 studies qualified majorities in voting procedures through reciprocal aggregation rules. In this framework, we prove that reciprocal aggregation rules of quasiarithmetic means generalize the simple majority, when individuals do not graduate their preferences (Proposition 2). Moreover, we show that this generalization is not valid when individuals graduate their preferences.

Notation and basic concepts. Given a set of alternatives $X \neq \emptyset$, a (strict) ordinary preference relation on $X$ is an asymmetric binary relation $P$ on $X[\forall x, y \in X \quad x P y \Rightarrow$ not $y P x]$. The indifference relation associated with $P$, denoted by $I$, reflects absence of preference: $x I y \Leftrightarrow$ 
not $x P y$ and not $y P x$. And the weak preference relation associated with $P$ is $P \cup I: x(P \cup$ $I) y \Leftrightarrow x P y$ or $x I y$.

A fuzzy subset $A$ of $X$ is defined through its membership function, $\mu_{A}: X \longrightarrow[0,1]$, where $\mu_{A}(x)$ is the membership grade of $x$ to $A$. The notion of fuzzy subset generalizes to the ordinary subset: a fuzzy subset $A$ of $X$ is ordinary if $\mu_{A}(X) \subseteq\{0,1\}$. According to the notion of fuzzy subset, a fuzzy binary relation on $X$ is a fuzzy subset of $X \times X$. The set of fuzzy binary relations on $X$ will be denoted by $\mathcal{R}(X)$. If $R$ is a fuzzy binary relation on $X=\left\{x_{1}, \ldots, x_{n}\right\}$ with membership function $\mu_{R}: X \times X \longrightarrow[0,1]$, we denote $r_{i j}=\mu_{R}\left(x_{i}, x_{j}\right)$.

The value of $r_{i j}$ has been interpreted in the literature in mainly two ways. For Orlovsky [32], Ovchinnikov [33] and Barrett-Pattanaik-Salles [2], among others, $r_{i j}$ is understood as the degree of certainty or confidence in the (strict or weak) preference of $x_{i}$ over $x_{j}$.

On the other hand, for Bezdek-Spillman-Spillman [5], Nurmi [31], Tanino [35] and Nakamura [30], among others, $r_{i j}$ denotes the intensity with which $x_{i}$ is preferred to $x_{j}$. Our paper is based on this second viewpoint. In this framework reciprocity is a common hypothesis: $r_{i j}+r_{j i}=1$ for all pair of alternatives $x_{i}, x_{j} \in X$. The set of reciprocal fuzzy binary relations on $X$ will be denoted by $\mathcal{R}_{r}(X)$.

We note that some authors, such as Bezdek-Spillman-Spillman [5] and Nurmi [31], among others, propose reciprocity with an exception: $r_{i i}=0$. These authors assume that $r_{i j}=\frac{1}{2}$ indicates indifference between $x_{i}$ and $x_{j}$. Since the alternative $x_{i}$ must be indifferent to itself, we have $r_{i i}=\frac{1}{2}$, just as happens under reciprocity. Thus, as the mentioned authors assert, $r_{i i}=0$ is a convention.

We also note that several factorizations into fuzzy strict preference and fuzzy indifference have appeared in the literature (see Dasgupta-Deb [14]) when $R \in \mathcal{R}(X)$ modelizes fuzzy weak preferences. However, under our interpretation of $r_{i j}$, reciprocal fuzzy binary relations do not need to be understood either as weak or as strict fuzzy preferences.

Now we justify that reciprocal fuzzy binary relations generalize ordinary preferences. Some authors, such as May [28] and Fishburn [21], use an index $d_{i j} \in\{-1,0,1\}$ to distinguish, in the ordinary case, between preference and indifference: $d_{i j}=1$, if $x_{i}$ is preferred to $x_{j} ; d_{i j}=0$, if $x_{i}$ is indifferent to $x_{j}$; and $d_{i j}=-1$, if $x_{j}$ is preferred to $x_{i}$. If we take

$$
r_{i j}=\frac{d_{i j}+1}{2}
$$

then we have $r_{i j}=1$ if $x_{i}$ is preferred to $x_{j} ; r_{i j}=\frac{1}{2}$ if $x_{i}$ is indifferent to $x_{j}$; and $r_{i j}=0$ if $x_{j}$ is preferred to $x_{i}$. Thus, $r_{i j} \in\left\{0, \frac{1}{2}, 1\right\}$ generates a reciprocal fuzzy binary relation. Consequently, ordinary preferences can be considered as a particular case of reciprocal fuzzy binary relations. 
In the sequel we suppose that fuzzy binary relations that represent preferences of individuals are reciprocal. These relations have a very important property: for each prefixed level of intensity $\alpha \geq \frac{1}{2}$ there exists an ordinary preference relation.

Given $\alpha \in[0,1)$, we define the ordinary binary relation of strict level $\alpha$ associated with $R$ as

$$
P_{\alpha}=\mu_{R}^{-1}((\alpha, 1])=\left\{\left(x_{i}, x_{j}\right) \in X \times X \mid r_{i j}>\alpha\right\},
$$

i.e., $x_{i} P_{\alpha} x_{j} \Leftrightarrow r_{i j}>\alpha$. We note that if $\alpha, \beta \in[0,1]$ are such that $\alpha \leq \beta$, then $P_{\beta} \subseteq P_{\alpha}$; i.e., for each pair of alternatives $x_{i}, x_{j} \in X: x_{i} P_{\beta} x_{j} \Rightarrow x_{i} P_{\alpha} x_{j}$. We note that every reciprocal fuzzy binary relation defines, in a natural way, a set of preference ordinary relations: if $R$ is a reciprocal fuzzy binary relation on $X$, then for each $\alpha \in\left[\frac{1}{2}, 1\right)$ the ordinary binary relation $P_{\alpha}$ is a preference relation. For $\alpha \in\left[\frac{1}{2}, 1\right)$, the indifference relation associated with $P_{\alpha}$, denoted by $I_{\alpha}$, is defined by $x_{i} I_{\alpha} x_{j} \Leftrightarrow 1-\alpha \leq r_{i j} \leq \alpha$. And the ordinary weak preference relation associated with $P_{\alpha}, P_{\alpha} \cup I_{\alpha}$, is defined by $x_{i}\left(P_{\alpha} \cup I_{\alpha}\right) x_{j} \Leftrightarrow r_{i j} \geq 1-\alpha$. So, for each chosen level $\alpha \in\left[\frac{1}{2}, 1\right), R$ has associated three ordinary relations: the preference $P_{\alpha}$, the indifference $I_{\alpha}$ and the weak preference $P_{\alpha} \cup I_{\alpha}$.

Fuzzy binary relations generalize ordinary binary relations. However, no reciprocal fuzzy binary relation is ordinary: from $r_{i i}=\frac{1}{2}$ for all $x_{i} \in X$, we have $\frac{1}{2} \in \mu_{R}(X \times X)$; hence, it is not verified $\mu_{R}(X \times X) \subseteq\{0,1\}$. Thus, we will distinguish between crisp preferences, where intensity levels are extreme $-0,1-$ or intermediate $-\frac{1}{2}-$, and gradual preferences, where other intensity levels appear.

So, individuals have crisp preferences when they do not graduate the intensity of preference among alternatives. In this case, for each pair of alternatives $x_{i}, x_{j} \in X$ :

1. If they prefer $x_{i}$ to $x_{j}$, they do so in an extreme way: $x_{i} P_{\alpha} x_{j}$ for all $\alpha \in\left[\frac{1}{2}, 1\right)$; i.e., $r_{i j}=1$ and then $r_{j i}=1-r_{i j}=0$.

2. Analogously, if they prefer $x_{j}$ to $x_{i}$, then $r_{j i}=1$, and $r_{i j}=1-r_{j i}=0$.

3. If they are indifferent between $x_{i}$ and $x_{j}$, then they do not prefer either alternative to the other: neither $x_{i} P_{\alpha} x_{j}$ nor $x_{j} P_{\alpha} x_{i}$, for all $\alpha \in\left[\frac{1}{2}, 1\right)$, i.e., $r_{i j} \leq \alpha$ and $r_{j i} \leq \alpha$ for all $\alpha \in\left[\frac{1}{2}, 1\right)$; hence, $1-\alpha \leq r_{i j} \leq \alpha$ for all $\alpha \in\left[\frac{1}{2}, 1\right)$, so $r_{i j}=r_{j i}=\frac{1}{2}$.

Consequently, $r_{i j} \in\left\{0, \frac{1}{2}, 1\right\}$ for all pair of alternatives $x_{i}, x_{j} \in X$.

On the contrary, an individual has gradual preferences when $r_{i j} \notin\left\{0, \frac{1}{2}, 1\right\}$, for some alternatives $x_{i}, x_{j} \in X$. 


\section{Neutral aggregation rules}

We consider $m$ individuals, $m \geq 2$, showing their preferences on a finite set of alternatives $X=\left\{x_{1}, \ldots, x_{n}\right\}, n \geq 2$, by means of reciprocal fuzzy binary relations.

\section{Definition 1}

An aggregation rule on $X$ is a sequence of functions $\mathcal{F}=\left\{F_{m}\right\}_{m \geq 2}$, such that $F_{m}: \mathcal{R}_{r}(X)^{m} \longrightarrow$ $\mathcal{R}(X)$ assigns to each profile of reciprocal fuzzy binary relations, $\left(R^{1}, \ldots, R^{m}\right) \in \mathcal{R}_{r}(X)^{m}$, a fuzzy binary relation, not necessarily reciprocal, $\bar{R}=F_{m}\left(R^{1}, \ldots, R^{m}\right) \in \mathcal{R}(X)$ called aggregate.

Notation. Given two alternatives $x_{i}, x_{j} \in X, r_{i j}^{k}=\mu_{R^{k}}\left(x_{i}, x_{j}\right)$ is the intensity level which individual $k$ prefers $x_{i}$ to $x_{j} ; \bar{r}_{i j}=\mu_{\bar{R}}\left(x_{i}, x_{j}\right)$ denotes the intensity level which alternative $x_{i}$ is collectivelly preferred to $x_{j}$, through the aggregate relation $\bar{R}$.

\section{Definition 2}

Let $\mathcal{F}$ be an aggregation rule on $X$.

1. $\mathcal{F}$ satisfies independence of irrelevant alternatives if for all pair of profiles $\left(R^{1}, \ldots, R^{m}\right)$, $\left(S^{1}, \ldots, S^{m}\right) \in \mathcal{R}_{r}(X)^{m}$ and all pair of alternatives $x_{i}, x_{j} \in X$ :

$$
\forall k \in\{1, \ldots, m\} \quad r_{i j}^{k}=s_{i j}^{k} \quad \Rightarrow \quad \bar{r}_{i j}=\bar{s}_{i j}
$$

2. $\mathcal{F}$ is neutral if for all pair of profiles $\left(R^{1}, \ldots, R^{m}\right),\left(S^{1}, \ldots, S^{m}\right) \in \mathcal{R}_{r}(X)^{m}$ and all alternatives $x_{i}, x_{j}, x_{p}, x_{q} \in X$ :

$$
\forall k \in\{1, \ldots, m\} \quad r_{i j}^{k}=s_{p q}^{k} \quad \Rightarrow \quad \bar{r}_{i j}=\bar{s}_{p q} .
$$

3. $\mathcal{F}$ is monotonic if for all pair of profiles $\left(R^{1}, \ldots, R^{m}\right),\left(S^{1}, \ldots, S^{m}\right) \in \mathcal{R}_{r}(X)^{m}$ and all pair of alternatives $x_{i}, x_{j} \in X$ :

$$
\forall k \in\{1, \ldots, m\} \quad r_{i j}^{k} \geq s_{i j}^{k} \quad \Rightarrow \quad \bar{r}_{i j} \geq \bar{s}_{i j}
$$

4. $\mathcal{F}$ is strictly monotonic if for all pair of profiles $\left(R^{1}, \ldots, R^{m}\right),\left(S^{1}, \ldots, S^{m}\right) \in \mathcal{R}_{r}(X)^{m}$ and all pair of alternatives $x_{i}, x_{j} \in X$ :

$$
\left(\forall k \in\{1, \ldots, m\} r_{i j}^{k} \geq s_{i j}^{k} \text { and } \exists l \in\{1, \ldots, m\} r_{i j}^{l}>s_{i j}^{l}\right) \quad \Rightarrow \quad \bar{r}_{i j}>\bar{s}_{i j} \text {. }
$$


5. $\mathcal{F}$ is anonymous if for all profile $\left(R^{1}, \ldots, R^{m}\right) \in \mathcal{R}_{r}(X)^{m}$ and all bijective function $\sigma:\{1, \ldots, m\} \longrightarrow\{1, \ldots, m\}:$

$$
F_{m}\left(R^{\sigma(1)}, \ldots, R^{\sigma(m)}\right)=F_{m}\left(R^{1}, \ldots, R^{m}\right) .
$$

6. $\mathcal{F}$ is unanimous if for all profile $\left(R^{1}, \ldots, R^{m}\right) \in \mathcal{R}_{r}(X)^{m}$, all pair of alternatives $x_{i}, x_{j} \in X$ and all $c \in[0,1]$ :

$$
\forall k \in\{1, \ldots, m\} r_{i j}^{k}=c \quad \Rightarrow \quad \bar{r}_{i j}=c .
$$

7. $\mathcal{F}$ is $\underline{\text { uniform }}$ if for all pair of profiles $\left(R^{1}, \ldots, R^{p}\right) \in \mathcal{R}_{r}(X)^{p},\left(S^{1}, \ldots, S^{q}\right) \in \mathcal{R}_{r}(X)^{q}$ and all pair of alternatives $x_{i}, x_{j} \in X$ :

$$
\left(\forall k \in\{1, \ldots, p\} \forall l \in\{1, \ldots, q\} r_{i j}^{k}=s_{i j}^{l}\right) \quad \Rightarrow \quad \bar{r}_{i j}=\bar{s}_{i j}
$$

8. $\mathcal{F}$ is reciprocal if for all profile $\left(R^{1}, \ldots, R^{m}\right) \in \mathcal{R}_{r}(X)^{m}$, the aggregate relation $\bar{R}=F_{m}\left(R^{1}, \ldots, R^{m}\right)$ is reciprocal.

\section{Remarks.}

1. Every neutral or monotonic aggregation rule satisfies independence of irrelevant alternatives.

2. Every strictly monotonic aggregation rule that satisfies independence of irrelevant alternatives is monotonic.

3. Every unanimous aggregation rule is uniform.

4. In monotonic aggregation rules, unanimity is equivalent to the fact that collective intensity of preference is found between minimal and maximal individual intensities.

In the following theorem we characterize neutral aggregation rules by means of functions. This result is related to another one in the crisp theory of welfare which says that there exists a social welfare function generating a given Paretian social welfare functional that satisfies the pairwise independence condition (see Mas-Colell-Whinston-Green [27, Proposition 22.D.1, p. 833]). This fact has been brought to our attention by an anonymous referee.

\section{Theorem 1}

If $\mathcal{F}$ is an aggregation rule on $X$, then the following conditions are equivalent:

1. $\mathcal{F}$ is neutral. 
2. For all $m \geq 2$ there exists a unique function $f_{m}:[0,1]^{m} \longrightarrow[0,1]$, such that for all pair of alternatives $x_{i}, x_{j} \in X: \bar{r}_{i j}=f_{m}\left(r_{i j}^{1}, \ldots, r_{i j}^{m}\right)$.

PROOF.

$1 \Rightarrow 2$ : By neutrality, $\mathcal{F}$ satisfies independence of irrelevant alternatives. So, given $m \geq 2$ and $x_{i}, x_{j} \in X$ with $i \neq j$, there exists a function $f_{m}^{i j}:[0,1]^{m} \longrightarrow[0,1]$, such that $\bar{r}_{i j}=$ $f_{m}^{i j}\left(r_{i j}^{1}, \ldots, r_{i j}^{m}\right)$, for each profile $\left(R^{1}, \ldots, R^{m}\right) \in \mathcal{R}_{r}(X)^{m}$. We now show that if $x_{p}, x_{q} \in X$ and $p \neq q$, then $f_{m}^{p q}=f_{m}^{i j}$. Given $\bar{a}=\left(a_{1}, \ldots, a_{m}\right) \in[0,1]^{m}$, there exist $\left(R^{1}, \ldots, R^{m}\right),\left(S^{1}, \ldots, S^{m}\right) \in$ $\mathcal{R}_{r}(X)^{m}$, such that $a_{k}=r_{i j}^{k}=s_{p q}^{k}$ for $k=1, \ldots, m$. By neutrality of $\mathcal{F}$, we have

$$
f_{m}^{i j}(\bar{a})=f_{m}^{i j}\left(r_{i j}^{1}, \ldots, r_{i j}^{m}\right)=\bar{r}_{i j}=\bar{s}_{p q}=f_{m}^{p q}\left(s_{p q}^{1}, \ldots, s_{p q}^{m}\right)=f_{m}^{p q}(\bar{a}) .
$$

Hence, there exists $f_{m}:[0,1]^{m} \longrightarrow[0,1]$, such that for all profile $\left(R^{1}, \ldots, R^{m}\right) \in \mathcal{R}_{r}(X)^{m}$ and all pair of alternatives $x_{i}, x_{j} \in X$, we have $\bar{r}_{i j}=f_{m}\left(r_{i j}^{1}, \ldots, r_{i j}^{m}\right)$, if $j \neq i$.

It remains to be shown that $\bar{r}_{i i}=f_{m}\left(\frac{1}{2}, \ldots, \frac{1}{2}\right)$. By reciprocity of the individual relations, we have $r_{i i}^{k}=\frac{1}{2}$ for all $k \in\{1, \ldots, m\}$. Given two alternatives $x_{p}, x_{q} \in X$ with $p \neq q$, let $\left(S^{1}, \ldots, S^{m}\right) \in \mathcal{R}_{r}(X)^{m}$ such that $s_{p q}^{k}=\frac{1}{2}$ for all $k \in\{1, \ldots, m\}$. Then, by neutrality of $\mathcal{F}$, we have $\bar{r}_{i i}=\bar{s}_{p q}=f_{m}\left(\frac{1}{2}, \ldots, \frac{1}{2}\right)$. Uniqueness of $f_{m}$ is guaranteed by construction.

$2 \Rightarrow 1:$ Let $\left(R^{1}, \ldots, R^{m}\right),\left(S^{1}, \ldots, S^{m}\right) \in \mathcal{R}_{r}(X)^{m}$ and $x_{i}, x_{j}, x_{p}, x_{q} \in X$, such that $r_{i j}^{k}=s_{p q}^{k}$ for all $k \in\{1, \ldots, m\}$. Then we have $\bar{r}_{i j}=f_{m}\left(r_{i j}^{1}, \ldots, r_{i j}^{m}\right)=f_{m}\left(s_{p q}^{1}, \ldots, s_{p q}^{m}\right)=\bar{s}_{p q}$.

\section{Notation.}

1. According to Theorem 1, every neutral aggregation rule $\mathcal{F}$ has an associated sequence of functions $\left\{f_{m}\right\}_{m \geq 2}$, where $f_{m}:[0,1]^{m} \longrightarrow[0,1]$, that we call auxiliary functions associated to $\mathcal{F}$.

2. Reciprocally, let $\left\{f_{m}\right\}_{m \geq 2}$ be a sequence of functions, where $f_{m}:[0,1]^{m} \longrightarrow[0,1]$. From this sequence can be defined a neutral aggregation rule $\mathcal{F}$, through $\bar{r}_{i j}=f_{m}\left(r_{i j}^{1}, \ldots, r_{i j}^{m}\right)$, for all pair of alternatives $x_{i}, x_{j} \in X$. We say that $\mathcal{F}$ is associated with $\left\{f_{m}\right\}_{m \geq 2}$. Obviously, these functions are the auxiliary functions associated with $\mathcal{F}$.

By means of Theorem 1, we can characterize some properties of a neutral aggregation rule $\mathcal{F}$, through conditions on the auxiliary functions associated with $\mathcal{F}$.

Notation. Let $\bar{a}, \bar{b} \in[0,1]^{m}$, where $\bar{a}=\left(a_{1}, \ldots, a_{m}\right), \bar{b}=\left(b_{1}, \ldots, b_{m}\right)$. With $\bar{a} \geq \bar{b}$ we denote $a_{i} \geq b_{i}$ for all $i \in\{1, \ldots, m\}$. With $\bar{a}>\bar{b}$ we understand $\bar{a} \geq \bar{b}$ and $a_{i}>b_{i}$ for some $i \in\{1, \ldots, m\}$. If $c \in[0,1]$ and $m \geq 2$, with $\bar{c}$ we denote $(c, \ldots, c) \in[0,1]^{m}$. 


\section{Corollary 1}

Let $\mathcal{F}$ be a neutral aggregation rule.

1. $\mathcal{F}$ is monotonic if and only if for all auxiliary function $f_{m}:[0,1]^{m} \longrightarrow[0,1]$ associated with $\mathcal{F}$ :

$$
\forall \bar{a}, \bar{b} \in[0,1]^{m} \quad\left(\bar{a} \geq \bar{b} \Rightarrow f_{m}(\bar{a}) \geq f_{m}(\bar{b})\right)
$$

2. $\mathcal{F}$ is strictly monotonic if and only if for all auxiliary function $f_{m}:[0,1]^{m} \longrightarrow[0,1]$ associated with $\mathcal{F}$ :

$$
\forall \bar{a}, \bar{b} \in[0,1]^{m} \quad\left(\bar{a}>\bar{b} \Rightarrow f_{m}(\bar{a})>f_{m}(\bar{b})\right)
$$

3. $\mathcal{F}$ is anonymous if and only if for all auxiliary function $f_{m}:[0,1]^{m} \longrightarrow[0,1]$ associated with $\mathcal{F}$ and all bijection $\sigma:\{1, \ldots, m\} \longrightarrow\{1, \ldots, m\}:$

$$
\forall \bar{a} \in[0,1]^{m} \quad f_{m}\left(a_{\sigma(1)}, \ldots, a_{\sigma(m)}\right)=f_{m}(\bar{a}) .
$$

4. $\mathcal{F}$ is unanimous if and only if for all auxiliary function $f_{m}:[0,1]^{m} \longrightarrow[0,1]$ associated with $\mathcal{F}$ :

$$
\forall c \in[0,1] \quad f_{m}(\bar{c})=c
$$

5. $\mathcal{F}$ is uniform if and only if for all pair of auxiliary functions $f_{p}:[0,1]^{p} \longrightarrow[0,1]$ and $f_{q}:[0,1]^{q} \longrightarrow[0,1]$ associated with $\mathcal{F}:$

$$
\forall c \in[0,1] \quad f_{p}(\bar{c})=f_{q}(\bar{c}) .
$$

6. $\mathcal{F}$ is reciprocal if and only if for all auxiliary function $f_{m}:[0,1]^{m} \longrightarrow[0,1]$ associated with $\mathcal{F}:$

$$
\forall \bar{a} \in[0,1]^{m} \quad f_{m}(\overline{1}-\bar{a})=1-f_{m}(\bar{a})
$$

Now we present several properties of the neutral aggregation rules that we use in our characterizations. These properties can be interpreted in the framework of the social choice theory.

Continuity means that small changes in individual intensities of preference produce small changes in the collective intensity of preference.

Additivity is a strong property used in the algebraic approach to social choice. It means that if the individual intensities of preference increase in some quantities, then the collective intensity of preference is the sum of both the collective intensity of preference corresponding to the initial individual intensities and also, the collective intensity of preference corresponding to the added 
quantities. We would like to remark that our additivity is related to another one used in utility theory.

Decomposability, a weaker property than additivity, means that the collective intensity of preference is the sum of the collective intensities of preference when, in each case, only one individual shows his/her preference and the others have a preference intensity of zero. This property is similar to a particular case of a very common property that appears in utility theory, called separability and also additivity. So, under some assumptions such as independence and essentiality, Debreu [15] proves that complete preorderings in product spaces can be represented by continuous and additive utility functions (see also Gorman [23]).

Lastly, associativity guarantees that each individual intensity of preference of a group can be substituted to its partial aggregation intensity without changing the collective intensity of preference.

\section{Definition 3}

Let $\mathcal{F}$ be a neutral aggregation rule.

1. $\mathcal{F}$ is continuous if all auxiliary functions associated with $\mathcal{F}$ are continuous.

2. $\mathcal{F}$ is additive if for all auxiliary function $f_{m}:[0,1]^{m} \longrightarrow[0,1]$ associated with $\mathcal{F}$ :

$$
\forall \bar{a}, \bar{b} \in[0,1]^{m} \quad\left(\bar{a}+\bar{b} \in[0,1]^{m} \Rightarrow f_{m}(\bar{a}+\bar{b})=f_{m}(\bar{a})+f_{m}(\bar{b})\right) .
$$

3. $\mathcal{F}$ is decomposable if for all auxiliary function $f_{m}:[0,1]^{m} \longrightarrow[0,1]$ associated with $\mathcal{F}$ :

$$
\forall \bar{a} \in[0,1]^{m} \quad f_{m}(\bar{a})=f_{m}\left(a_{1}, 0, \ldots, 0\right)+\cdots+f_{m}\left(0, \ldots, 0, a_{m}\right) .
$$

4. $\mathcal{F}$ is associative if for all auxiliary functions $f_{p}:[0,1]^{p} \longrightarrow[0,1]$ and $f_{p+q}:[0,1]^{p+q} \longrightarrow$ $[0,1]$ associated with $\mathcal{F}$, with $p \geq 2$ and $q \geq 0$ :

$$
\forall \bar{a} \in[0,1]^{p} \quad \forall \bar{b} \in[0,1]^{q} \quad f_{p+q}(\bar{a}, \bar{b})=f_{p+q}\left(f_{p}(\bar{a}), \ldots, f_{p}(\bar{a}), \bar{b}\right) .
$$

\section{Remarks.}

1. Every neutral and additive aggregation rule is decomposable and monotonic.

2. Every neutral, associative and strictly monotonic aggregation rule is unanimous.

3. Every auxiliary function $f_{m}:[0,1]^{m} \longrightarrow[0,1]$ of a decomposable neutral aggregation rule satisfies $f_{m}(\overline{0})=0$. 
4. There exist neutral and decomposable aggregation rules that are not monotonic and, hence, not additive either, for instance the one associated with the sequence of functions:

$$
f_{m}(\bar{a})=\frac{1}{m} \sum_{i=1}^{m} a_{i}\left(1-a_{i}\right), \quad m \geq 2 .
$$

5. There exist neutral and monotonic aggregation rules that are not decomposables and, hence, not additive either, for instance the one associated with the sequence of functions:

$$
f_{m}(\bar{a})=a_{1} \cdots \cdot a_{m}, \quad m \geq 2 .
$$

\section{Aggregation rules of the arithmetic means}

In Theorem 2 we characterize the neutral aggregation rules that are decomposable and anonymous. In these rules, collective intensity of preference is the arithmetic mean of the values assigned by a function of $[0,1]$ at the individual levels of intensity of preference. As properties are added to this class of aggregation rules, these properties are reflected in the functions of $[0,1]$.

\section{Definition 4}

Given a sequence of functions $\left\{\varphi_{m}\right\}_{m \geq 2}$, where $\varphi_{m}:[0,1] \longrightarrow[0,1]$ for all $m \geq 2$, we define the aggregation rule of the arithmetic mean associated with $\left\{\varphi_{m}\right\}_{m \geq 2}$ as the neutral aggregation rule $\mathcal{F}$ associated with $\left\{f_{m}\right\}_{m \geq 2}$, where $f_{m}:[0,1]^{m} \longrightarrow[0,1]$ is defined by

$$
f_{m}\left(a_{1}, \ldots, a_{m}\right)=\frac{\varphi_{m}\left(a_{1}\right)+\cdots+\varphi_{m}\left(a_{m}\right)}{m} .
$$

When the sequence of functions is constant, i.e. $\varphi_{m}=\varphi$ for all $m \geq 2$, we will say that $\mathcal{F}$ is the aggregation rule of the arithmetic mean associated with $\varphi$. In this case, if $\varphi$ is the identity function, we will say that $\mathcal{F}$ is the aggregation rule of the arithmetic mean.

\section{Theorem 2}

If $\mathcal{F}$ is a neutral aggregation rule, then the following conditions are equivalent:

1. $\mathcal{F}$ is decomposable and anonymous.

2. There exists a unique sequence of functions $\left\{\varphi_{m}\right\}_{m \geq 2}$, such that $\varphi_{m}(0)=0$ for all $m \geq 2$ and $\mathcal{F}$ is the aggregation rule of the arithmetic mean associated with $\left\{\varphi_{m}\right\}_{m \geq 2}$. 
Proof.

$1 \Rightarrow 2$ : Fixed $m$, let $\varphi_{m}:[0,1] \longrightarrow[0,1]$ be the function defined by $\varphi_{m}(c)=f_{m}(\bar{c})$ for all $c \in[0,1]$, where $f_{m}:[0,1]^{m} \longrightarrow[0,1]$ is the corresponding auxiliary function associated with $\mathcal{F}$. Then, $\varphi_{m}(0)=f_{m}(\overline{0})=0$. Moreover, for all $\bar{a} \in[0,1]^{m}$, it satisfies

$$
\begin{aligned}
& m f_{m}(\bar{a})=m f_{m}\left(a_{1}, 0, \ldots, 0\right)+\cdots+m f_{m}\left(0, \ldots, 0, a_{m}\right)= \\
& =f_{m}\left(\bar{a}_{1}\right)+\cdots+f_{m}\left(\bar{a}_{m}\right)=\varphi_{m}\left(a_{1}\right)+\cdots+\varphi_{m}\left(a_{m}\right) .
\end{aligned}
$$

In order to justify uniqueness, let $\left\{\psi_{m}\right\}_{m \geq 2}$ be a sequence of functions that satisfies what is required. Then, for all $c \in[0,1]$ we have

$$
\varphi_{m}(c)=f_{m}(\bar{c})=\frac{1}{m} m \psi_{m}(c)=\psi_{m}(c) .
$$

$2 \Rightarrow 1$ : For all auxiliary function $f_{m}:[0,1]^{m} \longrightarrow[0,1]$ associated with $\mathcal{F}$ and all $\bar{a} \in[0,1]^{m}$, it satisfies

$$
\begin{aligned}
& f_{m}\left(a_{1}, 0, \ldots, 0\right)+\cdots+f_{m}\left(0, \ldots, 0, a_{m}\right)= \\
& =\frac{1}{m}\left(\varphi_{m}\left(a_{1}\right)+\varphi_{m}(0)+\cdots+\varphi_{m}(0)\right)+\cdots+\frac{1}{m}\left(\varphi_{m}(0)+\cdots+\varphi_{m}(0)+\varphi_{m}\left(a_{m}\right)\right)= \\
& =\frac{1}{m}\left(\varphi_{m}\left(a_{1}\right)+\cdots+\varphi_{m}\left(a_{m}\right)\right)=f_{m}(\bar{a}) .
\end{aligned}
$$

Obviously, $\mathcal{F}$ is anonymous.

\section{Corollary 2}

If $\mathcal{F}$ is a neutral aggregation rule, then the following conditions are equivalent:

1. $\mathcal{F}$ is decomposable, anonymous and uniform.

2. There exists a unique function $\varphi$, with $\varphi(0)=0$, such that $\mathcal{F}$ is the aggregation rule of the arithmetic mean associated with $\varphi$.

Proof.

$1 \Rightarrow 2$ : If in the proof of Theorem 2 we consider $\mathcal{F}$ uniform, we have $\varphi_{p}(c)=f_{p}(\bar{c})=f_{q}(\bar{c})=$ $\varphi_{q}(c)$ for all $p, q \geq 2$ and all $c \in[0,1]$. Hence, there exists $\varphi$ such that $\varphi_{m}=\varphi$ for all $m \geq 2$. Consequently, $\mathcal{F}$ is the aggregation rule of the arithmetic mean associated with $\varphi$.

$2 \Rightarrow 1$ : Obvious. 


\section{Corollary 3}

If $\mathcal{F}$ is a neutral aggregation rule, then the following conditions are equivalent:

1. $\mathcal{F}$ is decomposable, unanimous and anonymous.

2. $\mathcal{F}$ is the aggregation rule of the arithmetic mean.

Proof.

$1 \Rightarrow 2$ : If in the proof of Theorem 2 we consider $\mathcal{F}$ unanimous, we have $\varphi_{m}(c)=f_{m}(\bar{c})=c$ for all $m \geq 2$ and $c \in[0,1]$, i.e. $\varphi_{m}$ is the identity function for all $m \geq 2$. Hence, $\mathcal{F}$ is the aggregation rule of the arithmetic mean.

$2 \Rightarrow 1$ : Obvious.

\section{Remarks.}

1. A similar result to Corollary 3 has been proved on topological vectorial spaces of alternative sets, by Candeal-Induráin-Uriarte [11], outside of the fuzzy preferences framework.

2. When the set of alternatives has a group operation, Candeal-Induráin [12, Theorem 1] proved a result based in Chichilnisky-Heal [13]: every group Chichilnisky $n$-rule (group homomorphism, anonymous and unanimous aggregation rule) is a convex mean (a generalization of the arithmetic mean for groups).

3. The aggregation rule of the arithmetic mean satisfies all the properties considered in the paper (Definitions 2 and 3).

\section{Corollary 4}

If $\mathcal{F}$ is a neutral aggregation rule, then the following conditions are equivalent:

1. $\mathcal{F}$ is additive and anonymous.

2. There exists a unique sequence of functions $\left\{\varphi_{m}\right\}_{m \geq 2}$, that satisfies $\varphi_{m}(a+b)=\varphi_{m}(a)+$ $\varphi_{m}(b)$ for all $m \geq 2$ and $a, b \in[0,1]$ with $a+b \in[0,1]$, such that $\mathcal{F}$ is the aggregation rule of the arithmetic mean associated with $\left\{\varphi_{m}\right\}_{m \geq 2}$. 
Proof. We consider Theorem 2 and its proof.

$1 \Rightarrow 2$ : We take into account that additive aggregation rules are decomposable. Let $a, b \in[0,1]$ be such that $a+b \in[0,1]$. Then $\overline{a+b} \in[0,1]^{m}$ and

$$
\varphi_{m}(a+b)=f_{m}(\overline{a+b})=f_{m}(\bar{a}+\bar{b})=f_{m}(\bar{a})+f_{m}(\bar{b})=\varphi_{m}(a)+\varphi_{m}(b) .
$$

$2 \Rightarrow 1$ : From additivity of $\varphi_{m}$, we have $\varphi_{m}(0)=0$ and additivity of $\mathcal{F}$. By Theorem $2, \mathcal{F}$ is anonymous.

\section{Corollary 5}

If $\mathcal{F}$ is a neutral aggregation rule, then the following conditions are equivalent:

1. $\mathcal{F}$ is decomposable, anonymous and monotonic (strictly monotonic).

2. There exists a unique sequence of increasing (strictly increasing) functions $\left\{\varphi_{m}\right\}_{m \geq 2}$, with $\varphi_{m}(0)=0$ for all $m \geq 2$, such that $\mathcal{F}$ is the aggregation rule of the arithmetic mean associated with $\left\{\varphi_{m}\right\}_{m \geq 2}$.

Proof. We consider Theorem 2 and its proof. We prove the equivalence for monotonicity (for strict monotonicity the proof is analogous).

$1 \Rightarrow 2$ : We need only show that $\varphi_{m}$ is increasing. Let $a, b \in[0,1]$ such that $a \geq b$; then $\bar{a} \geq \bar{b}$ and $\varphi_{m}(a)=f_{m}(\bar{a}) \geq f_{m}(\bar{b})=\varphi_{m}(b)$.

$2 \Rightarrow 1$ : The only thing that needs proof is that $\mathcal{F}$ is monotonic. Let $\bar{a}, \bar{b} \in[0,1]^{m}$ such that $\bar{a} \geq \bar{b}$; then $a_{i} \geq b_{i}$ and $\varphi_{m}\left(a_{i}\right) \geq \varphi_{m}\left(b_{i}\right)$ for all $i \in\{1, \ldots, m\}$. Hence

$$
f_{m}(\bar{a})=\frac{1}{m} \sum_{i=1}^{m} \varphi_{m}\left(a_{i}\right) \geq \frac{1}{m} \sum_{i=1}^{m} \varphi_{m}\left(b_{i}\right)=f_{m}(\bar{b}) .
$$

Two inmediate consequences of Theorem 2 are the following corollaries.

\section{Corollary 6}

If $\mathcal{F}$ is a neutral aggregation rule, then the following conditions are equivalent:

1. $\mathcal{F}$ is decomposable, anonymous and reciprocal. 
2. There exists a unique sequence of functions $\left\{\varphi_{m}\right\}_{m \geq 2}$, with $\varphi_{m}(0)=0$ and $\varphi_{m}(1-a)=$ $1-\varphi_{m}(a)$ for all $m \geq 2$ and all $a \in[0,1]$, such that $\mathcal{F}$ is the aggregation rule of the arithmetic mean associated with $\left\{\varphi_{m}\right\}_{m \geq 2}$.

\section{Corollary 7}

If $\mathcal{F}$ is a neutral aggregation rule, then the following conditions are equivalent:

1. $\mathcal{F}$ is decomposable, anonymous and continuous.

2. There exists a unique sequence of continuous functions $\left\{\varphi_{m}\right\}_{m \geq 2}$, with $\varphi_{m}(0)=0$ for all $m \geq 2$, such that $\mathcal{F}$ is the aggregation rule of the arithmetic mean associated with $\left\{\varphi_{m}\right\}_{m \geq 2}$.

\section{Aggregation rules of the quasiarithmetic means}

Firstly we introduce order automorphisms of $[0,1]$ and we deduce several consequences.

\section{Definition 5}

A function $\varphi:[0,1] \longrightarrow[0,1]$ is an order automorphism if it is bijective and increasing.

\section{Lemma 1}

If $\varphi:[0,1] \longrightarrow[0,1]$ is an order automorphism, then:

1. $\varphi$ is strictly increasing.

2. $\varphi^{-1}$ is an order automorphism.

3. $\varphi(0)=0$ and $\varphi(1)=1$.

4. $\varphi$ is continuous.

5. $\forall a \in[0,1] \quad \varphi(1-a)=1-\varphi(a) \quad \Leftrightarrow \quad \forall a \in[0,1] \quad \varphi^{-1}(1-a)=1-\varphi^{-1}(a)$.

Proof 
1. Given $a, b \in[0,1]$, such that $a<b$, we have $\varphi(a) \neq \varphi(b)$ and $\varphi(a) \leq \varphi(b)$; hence $\varphi(a)<\varphi(b)$.

2. Obviously $\varphi^{-1}$ is bijective. If $\varphi^{-1}$ is not increasing, there exist $a, b \in[0,1]$, such that $a \leq b$ and $\varphi^{-1}(a)>\varphi^{-1}(b)$. Since $\varphi$ is strictly increasing, we have $a=\varphi\left(\varphi^{-1}(a)\right)>$ $\varphi\left(\varphi^{-1}(b)\right)=b$, which violates $a \leq b$.

3. If $\varphi(0) \neq 0$, then there exists $a \in(0,1]$ such that $\varphi(a)=0$, contrary to $\varphi$ be increasing. Analogously, we obtain $\varphi(1)=1$.

4. We show that $\varphi$ is continuous in an arbitrary $a_{0} \in(0,1)$. Given $\varepsilon>0$, let $a_{1}, a_{2} \in[0,1]$ such that $\varphi\left(a_{1}\right)=\max \left\{0, \varphi\left(a_{0}\right)-\varepsilon\right\}, \varphi\left(a_{2}\right)=\min \left\{1, \varphi\left(a_{0}\right)+\varepsilon\right\}$. Since $\varphi^{-1}$ is strictly increasing, we have $a_{1}<a_{0}<a_{2}$. If $\delta=\min \left\{a_{0}-a_{1}, a_{2}-a_{0}\right\}$, since $\varphi$ is strictly increasing, for all $a \in[0,1]$ we have

$$
\left|a-a_{0}\right|<\delta \Rightarrow\left|\varphi(a)-\varphi\left(a_{0}\right)\right|<\varepsilon .
$$

It can similarly be established the continuity of $\varphi$ in 0 and 1.

5. It is sufficient to prove that if the statement is true for an order automorphism $\varphi$, then it is also true for $\varphi^{-1}$. We prove $\varphi^{-1}(1-a)=1-\varphi^{-1}(a)$ for all $a \in[0,1]$. If $b=\varphi^{-1}(1-a)$, we have $1-a=\varphi(b)$, i.e. $a=1-\varphi(b)=\varphi(1-b)$. Hence,

$$
1-\varphi^{-1}(a)=1-\varphi^{-1}(\varphi(1-b))=1-(1-b)=b=\varphi^{-1}(1-a) .
$$

Now we introduce the aggregation rules of the quasiarithmetic means associated with order automorphisms. The result of Kolmogoroff [26] and Nagumo [29] allows us to characterize this class of aggregation rules in a natural way from the point of view of collective decision making.

\section{Definition 6}

Given an order automorphism $\varphi:[0,1] \longrightarrow[0,1]$, we define the aggregation rule of the quasiarith$\underline{\text { metic mean associated with } \varphi}$ as the neutral aggregation rule associated with the sequence of functions $\left\{f_{m}\right\}_{m \geq 2}$, where $f_{m}:[0,1]^{m} \longrightarrow[0,1]$ is definided by

$$
f_{m}\left(a_{1}, \ldots, a_{m}\right)=\varphi^{-1}\left(\frac{\varphi\left(a_{1}\right)+\cdots+\varphi\left(a_{m}\right)}{m}\right) .
$$

Obviously, the aggregation rule of the quasiarithmetic mean associated with the identity is the aggregation rule of the arithmetic mean. Using a similar idea to the proof of Proposition 1, it can be proved that the aggregation rule of the quasiarithmetic mean associated with $\varphi$ is the aggregation rule of the arithmetic mean only when $\varphi$ is the identity. 
Theorem 3 (Kolmogoroff - Nagumo, 1930)

If $\mathcal{F}$ is a neutral aggregation rule, then the following conditions are equivalent:

1. $\mathcal{F}$ is an aggregation rule of some quasiarithmetic mean.

2. $\mathcal{F}$ is anonymous, unanimous, continuous, strictly monotonic and associative.

Proof.

$1 \Rightarrow 2$ : The verification is purely mechanical.

$2 \Rightarrow 1$ : It is an inmediate consequence of Kolmogoroff [26] and Nagumo [29].

\section{Remarks.}

1. In Theorem 3, unanimity is redundant, since it is consequence of associativity and strict monotonicity.

2. The aggregation rules of quasiarithmetic means are not necessarily reciprocal; for instance, if $\varphi(a)=a^{2}$ for all $a \in[0,1]$, we have $f_{2}(1-0.6,1-0.8) \neq 1-f_{2}(0.6,0.8)$.

In the following proposition we give a necessary and sufficient condition for reciprocity in aggregation rules of the quasiarithmetic means.

\section{Proposition 1}

If $\mathcal{F}$ is the aggregation rule of the quasiarithmetic mean associated with $\varphi$, then

$$
\mathcal{F} \text { is reciprocal } \Leftrightarrow \forall a \in[0,1] \quad \varphi(1-a)=1-\varphi(a) .
$$

Proof.

$\Rightarrow)$ We prove the property for $\varphi^{-1}$. We first prove the property for rational numbers. For $a=0$ and $a=1$ it is obviousus. If $a \in(0,1)$ is rational, there exist $m, p \in \mathbb{N}$ with $\operatorname{gcd}(m, p)=1$, such that $a=\frac{p}{m}$, with $p<m$ and $m \geq 2$. If we consider $\bar{b}=\left(b_{1}, \ldots, b_{m}\right) \in[0,1]^{m}$ such that

$$
b_{i}= \begin{cases}1 & \forall i \in\{1, \ldots, p\} \\ 0 & \forall i \in\{p+1, \ldots, m\}\end{cases}
$$


then, we have

$$
f_{m}(\bar{b})=\varphi^{-1}\left(\frac{1}{m}\left(\sum_{i=1}^{p} \varphi(1)+\sum_{i=p+1}^{m} \varphi(0)\right)\right)=\varphi^{-1}\left(\frac{1}{m} p \varphi(1)\right)=\varphi^{-1}\left(\frac{p}{m}\right) .
$$

On the other hand,

$$
\begin{aligned}
& f_{m}(\bar{b})=f_{m}(\overline{1}-(\overline{1}-\bar{b}))=1-f_{m}(\overline{1}-\bar{b})=1-\varphi^{-1}\left(\frac{1}{m}\left(\sum_{i=1}^{p} \varphi(0)+\sum_{i=p+1}^{m} \varphi(1)\right)\right)= \\
& =1-\varphi^{-1}\left(\frac{1}{m}(m-p) \varphi(1)\right)=1-\varphi^{-1}\left(\frac{m-p}{m}\right)=1-\varphi^{-1}\left(1-\frac{p}{m}\right) .
\end{aligned}
$$

Hence, $\varphi^{-1}\left(\frac{p}{m}\right)=1-\varphi^{-1}\left(1-\frac{p}{m}\right)$, i.e.,

$$
\varphi^{-1}(1-a)=\varphi^{-1}\left(1-\frac{p}{m}\right)=1-\varphi^{-1}\left(\frac{p}{m}\right)=1-\varphi^{-1}(a) .
$$

For irrationals, it is sufficient take into account continuity of $\varphi^{-1}$ and density of rationals in $\mathbb{R}$. Given $a \in(0,1)$ irrational, there exists a sequence of rationals of $[0,1],\left\{a_{n}\right\}_{n=1}^{\infty}$, that converges to $a$. Then, $\lim _{n \rightarrow \infty}\left(1-a_{n}\right)=1-a$ and, by continuity of $\varphi^{-1}$, we have

$$
\varphi^{-1}(1-a)=\lim _{n \rightarrow \infty} \varphi^{-1}\left(1-a_{n}\right)=\lim _{n \rightarrow \infty}\left(1-\varphi^{-1}\left(a_{n}\right)\right)=1-\lim _{n \rightarrow \infty} \varphi^{-1}\left(a_{n}\right)=1-\varphi^{-1}(a) .
$$

$\Leftarrow)$ Let $\bar{a} \in[0,1]^{m}$. By properties of $\varphi$, we have:

$$
\begin{aligned}
& f_{m}(\overline{1}-\bar{a})=\varphi^{-1}\left(\frac{1}{m} \sum_{i=1}^{m} \varphi\left(1-a_{i}\right)\right)=\varphi^{-1}\left(\frac{1}{m} \sum_{i=1}^{m}\left(1-\varphi\left(a_{i}\right)\right)\right)= \\
& =\varphi^{-1}\left(1-\frac{1}{m} \sum_{i=1}^{m} \varphi\left(a_{i}\right)\right)=1-\varphi^{-1}\left(\frac{1}{m} \sum_{i=1}^{m} \varphi\left(a_{i}\right)\right)=1-f_{m}(\bar{a}) .
\end{aligned}
$$

\section{Qualified majorities}

We now analize, by means of reciprocal fuzzy binary relations, voting procedures by qualified majorities, both when individuals have crisp or gradual preferences.

Given a reciprocal aggregation rule $\mathcal{F}$, for every finite number of individuals $m$, the function $F_{m}: \mathcal{R}_{r}(X)^{m} \longrightarrow \mathcal{R}(X)$ assigns a reciprocal fuzzy binary relation, the aggregate $\bar{R}=$ $F_{m}\left(R^{1}, \ldots, R^{m}\right)$, to each profile of reciprocal fuzzy binary relations $\left(R^{1}, \ldots, R^{m}\right) \in \mathcal{R}_{r}(X)^{m}$. We denote by $\mathcal{P}(X)$ the set of ordinary preference relations on $X$. We know that for each $\alpha \in\left[\frac{1}{2}, 1\right)$ we have a function $\Pi_{\alpha}: \mathcal{R}_{r}(X) \longrightarrow \mathcal{P}(X)$ that assigns to $\bar{R}$ the ordinary relation of preference, $\Pi_{\alpha}(\bar{R})=\bar{P}_{\alpha}=\left\{\left(x_{i}, x_{j}\right) \in X \times X \mid \bar{r}_{i j}>\alpha\right\}$. This relation shows which alternatives are collectivelly preferred to others with a intensity level greater to $\alpha$. 


\section{Definition 7}

Let $\mathcal{F}$ be a reciprocal aggregation rule on $X, m$ individuals and $\alpha \in\left[\frac{1}{2}, 1\right)$. We define

$$
F_{m}^{\alpha}=\Pi_{\alpha} \circ F_{m}: \mathcal{R}_{r}(X)^{m} \longrightarrow \mathcal{P}(X)
$$

the $\alpha$-majority aggregation function associated with $\mathcal{F}$ and $m$.

$$
\begin{array}{ccccc}
\mathcal{R}_{r}(X)^{m} & \stackrel{F_{m}}{\longmapsto} & \mathcal{R}_{r}(X) & \stackrel{\Pi_{\alpha}}{\longmapsto} & \mathcal{P}(X) \\
\left(R^{1}, \ldots, R^{m}\right) & \longmapsto & \bar{R} & \longmapsto & \bar{P}_{\alpha}
\end{array}
$$

Remark. Using qualified majorities for collective decision making, corresponding to increasing levels of $\alpha$, some considerations are taken into account:

- A greater support from individuals is necessary for defeating one alternative to another, the more support being needed the greater $\alpha$ is.

- The voting procedures are less decisive, since collective indifference appears more frequently $\left(x_{i} \bar{I}_{\alpha} x_{j} \Leftrightarrow 1-\alpha \leq \bar{r}_{i j} \leq \alpha\right)$.

- It decreases the possibility of existence of cycles in collective preferences (voting paradox). Since there are less alternatives collectively preferred to other, it is more possible that collective preference will be acyclic. Consequently, more non-dominated alternatives in all non-empty subsets will exist (see Ferejohn-Grether [20, Th. 1]).

It is clear that all reciprocal aggregation rules, particularly these ones studied in this paper, permit establish voting procedures with different levels of qualification.

Now we justify that reciprocal aggregation rules of the quasiarithmetic means generalize the simple majority when individuals have crisp preferences. In this case, the 0.5 - majority aggregation function associated with every reciprocal aggregation rule of a quasiarithmetic mean provides the same results as the simple majority.

\section{Proposition 2}

Let $\mathcal{F}$ be the quasiarithmetic mean aggregation rule on $X$ associated with $\varphi$. If $\mathcal{F}$ is reciprocal and all individuals have crisp preferences, then $F_{m}^{0.5}$ coincide with simple majority, for all $m \geq 2$.

Proof. We suppose $m$ individuals with crisp preferences in $X$ :

$$
\forall k \in\{1, \ldots, m\} \quad \forall x_{i}, x_{j} \in X \quad r_{i j}^{k} \in\{0,0.5,1\} .
$$


Firstly, we note $\varphi(0)=0, \varphi(1)=1$ and, by reciprocity, $\varphi(0.5)=0.5$. Moreover, we have:

$$
\begin{aligned}
& m=\#\left\{k \mid x_{i} P_{0.5}^{k} x_{j}\right\}+\#\left\{k \mid x_{i} I_{0.5}^{k} x_{j}\right\}+\#\left\{k \mid x_{j} P_{0.5}^{k} x_{i}\right\}= \\
& =\#\left\{k \mid r_{i j}^{k}=1\right\}+\#\left\{k \mid r_{i j}^{k}=0.5\right\}+\#\left\{k \mid r_{i j}^{k}=0\right\} .
\end{aligned}
$$

Now, let $\bar{P}_{s}$ the aggregate preference relation corresponding to simple majority, i.e. $x_{i} \bar{P}_{s} x_{j} \Leftrightarrow$ $\#\left\{k \mid r_{i j}^{k}=1\right\}>\#\left\{k \mid r_{i j}^{k}=0\right\}$, and $\bar{P}_{0.5}=F_{m}^{0.5}\left(R^{1}, \ldots, R^{m}\right)$.

Then we have:

$$
\begin{aligned}
& x_{i} \bar{P}_{0.5} x_{j} \Leftrightarrow \bar{r}_{i j}>0.5 \Leftrightarrow \varphi^{-1}\left(\frac{1}{m} \sum_{k=1}^{m} \varphi\left(r_{i j}^{k}\right)\right)>0.5 \Leftrightarrow \frac{1}{m} \sum_{k=1}^{m} \varphi\left(r_{i j}^{k}\right)>\varphi(0.5)=0.5 \Leftrightarrow \\
& \Leftrightarrow \frac{1}{m}\left(\#\left\{k \mid r_{i j}^{k}=1\right\} \cdot 1+\#\left\{k \mid r_{i j}^{k}=0.5\right\} \cdot 0.5+\#\left\{k \mid r_{i j}^{k}=0\right\} \cdot 0\right)>0.5 \Leftrightarrow \\
& \Leftrightarrow \#\left\{k \mid r_{i j}^{k}=1\right\}+\#\left\{k \mid r_{i j}^{k}=0.5\right\} \cdot 0.5>0.5 \cdot m \Leftrightarrow \\
& \Leftrightarrow \#\left\{k \mid r_{i j}^{k}=1\right\}>0.5 \cdot\left(m-\#\left\{k \mid r_{i j}^{k}=0.5\right\}\right) \Leftrightarrow \\
& \Leftrightarrow \#\left\{k \mid r_{i j}^{k}=1\right\}>0.5 \cdot\left(\#\left\{k \mid r_{i j}^{k}=1\right\}+\#\left\{k \mid r_{i j}^{k}=0\right\}\right) \Leftrightarrow \\
& \Leftrightarrow \#\left\{k \mid r_{i j}^{k}=1\right\}>\#\left\{k \mid r_{i j}^{k}=0\right\} \Leftrightarrow x_{i} \bar{P}_{s} x_{j} .
\end{aligned}
$$

Remark. Let 5 individuals with gradual preferences on two alternatives $x_{1}$ and $x_{2}$ :

$$
\begin{aligned}
& r_{12}^{1}=r_{12}^{2}=r_{12}^{3}=0.6 \quad\left(r_{21}^{1}=r_{21}^{2}=r_{21}^{3}=0.4\right) \\
& r_{12}^{4}=r_{12}^{5}=0.1 \quad\left(r_{21}^{4}=r_{21}^{5}=0.9\right) .
\end{aligned}
$$

If individuals have to show their preferences in a crisp way, through $P_{0.5}$, then $x_{1}$ defeats $x_{2}$ by simple majority, since there are 3 individuals that prefer $x_{1}$ to $x_{2}$.

However, if individuals can show their preferences in their natural gradual way, then using the aggregation rule of the arithmetic mean, through $\bar{P}_{0.5}, x_{2}$ defeats $x_{1}$, since

$$
\bar{r}_{21}=\sum_{k=1}^{5} \frac{r_{21}^{k}}{5}=\frac{3 \cdot 0.4+2 \cdot 0.9}{5}=0.6>0.5
$$

We note $x_{2} \bar{P}_{\alpha} x_{1}$ for all $\alpha \in[0.5,0.6)$; if $\alpha \in[0.6,1)$ we have $x_{2} \bar{I}_{\alpha} x_{1}$. Moreover, for not $\alpha \in[0.5,1)$ we obtain $x_{1} \bar{P}_{\alpha} x_{2}$.

We can conclude that the result obtained applying simple majority when individuals only show their preferred alternatives can be different to that obtained applying an aggregation rule of some quasiarithmetic mean, when individuals show intensity levels of their preferences. 


\section{References}

[1] Aumann G (1943) "ber Rame mit Mittelbildungen": Math. Ann. 119: 210-215.

[2] Barrett CR - Pattanaik PK - Salles M (1986) "On the structure of fuzzy social welfare functions": Fuzzy Sets and Systems 19: 1-10.

[3] Barrett CR - Pattanaik PK - Salles M (1992) "Rationality and aggregation of preferences in an ordinally fuzzy framework": Fuzzy Sets and Systems 49: 9-14.

[4] Bellman RE - Zadeh LA (1970) "Decision-making in a fuzzy environment": Management Science 17: 141-164.

[5] Bezdek JC - Spillman B - Spillman R (1978) "A fuzzy relation space for group decision theory: an application": Fuzzy Sets and Systems 1: 255-268.

[6] Bezdek JC - Spillman B - Spillman R (1979) "Fuzzy relation spaces for group decision theory: an application": Fuzzy Sets and Systems 2: 5-14.

[7] Billot A (1991) "Aggregation of preferences: the fuzzy case": Theory and Decision 30: 51-93.

[8] Blin JM - Whinston AB (1974) "Fuzzy sets and social choice": Journal of Cybernetics 3: $28-36$

[9] BLIN JM (1974) "Fuzzy relation in group decision theory": Journal of Cybernetics 4: 17-22.

[10] Bullen PS - Mitrinović DS - VAsić PM (1988) Means and Their Inequalities. Reidel, Dordrecht.

[11] Candeal JC - Induráin E - Uriarte JR (1992) "Some issues related to the topological aggregation of preferences": Social Choice and Welfare 9: 213-227.

[12] Candeal JC - Induráin E (1995) "Aggregation of preferences from algebraic models on groups": Social Choice and Welfare 12: 165-173.

[13] Chichilnisky G - Heal G (1983) "Necessary and sufficient conditions for a resolution of the social choice paradox": Journal of Economic Theory 31: 68-87.

[14] Dasgupta M - Deb R "Factoring fuzzy transitivity": Fuzzy Sets and Systems: forthcoming.

[15] Debreu G (1960) "Topological methods in cardinal utility theory": In ArRow KJ - KARLIN S - Suppes P (eds.) (1960) Mathematical Methods in the Social Sciences, 1959: 16-26. Stanford University Press, Stanford. 
[16] Dubois D - Koning JL (1991) "Social choice axioms for fuzzy set aggregation": Fuzzy Sets and Systems 43: 257-274.

[17] Dubois D - Prade H (1985) "A review of fuzzy set aggregation connectives": Information Sciences 36: 85-121.

[18] Eckmann B (1954) "Rame mit Mittelbildungen": Comentari Mathematici Helvetici 28: 329-340.

[19] Eckmann B - Ganea T - Hilton PJ (1962) "Generalized means": Studies in Mathematical Analysis and Related Topics. Stanford University Press, Stanford: 82-92.

[20] Ferejohn JA - Grether DM (1974) "On a class of rational social decisions procedures": Journal of Economic Theory 8: 471-482.

[21] FishbuRn PC (1973) The Theory of Social Choice. Princeton University Press, Princeton.

[22] Fodor J - Roubens M (1994) Fuzzy Preference Modelling and Multicriteria Decision Support. Kluwer Academic Publishers, Dordrecht.

[23] Gorman W.M. (1968) "The structure of utility functions": Review of Economic Studies 35: $367-390$.

[24] Horvath CD "On the topological social choice problem": Social Choice and Welfare: forthcoming.

[25] Horvath CD (1999) "Some aspects of the mathematical background of the topological social choice problem": Mimeo.

[26] Kolmogoroff A (1930) "Sur la notion de la moyenne": Atti della R. Academia Nazionale del Lincei. Rendiconti della Classe di Scienze Fisiche, Mathematiche e Naturali (6) 12: 388391.

[27] Mas-Colell A - Whinston MD - Green JR (1995) Microeconomic Theory. Oxford University Press, New York.

[28] MAY KO (1952) "A set of independent necessary and sufficcient conditions for simple majority decision": Econometrica 20: 680-684.

[29] Nagumo M (1930) "ber eine Klasse der Mittelwerte": Japanese Journal of Mathematics 7: 71-79. 
[30] NAKAmURA K (1992) "On the nature of intransitivity in human preferential judgements": In Novák V - RAmík J et al (eds.) (1992) Fuzzy Approach to Reasoning and DecisionMaking: 147-162. Kluwer Academic Publishers, Dordrecht.

[31] Nurmi H (1981) "Approaches to collective decision making with fuzzy preference relations": Fuzzy Sets and Systems 6: 249-259.

[32] Orlovsky, SA (1978) "Decision-making with a fuzzy preference relation": Fuzzy Sets and Systems 1: 155-167.

[33] Ovchinnikov SV (1981) "Structure of fuzzy binary relations": Fuzzy Sets and Systems 6: 169-195.

[34] Ovchinnikov SV (1990) "Means and social welfare function in fuzzy binary relation spaces": In KACPRzyK J - Fedrizzi M (eds.) (1990) Multiperson Decision Making Using Fuzzy Sets and Possibility Theory: 143-154. Kluwer Academic Publishers, Dordrecht.

[35] Tanino T (1984) "Fuzzy preference orderings in group decision making": Fuzzy Sets and Systems 12: 117-131.

[36] Yager RR (1988) "Ordered weighted averaging aggregation operators in multi-criteria decision making": IEEE Trans. on Systems, Man and Cybernetics 8: 183-190.

[37] Yager RR (1994) "Aggregation operators and fuzzy systems modeling": Fuzzy Sets and Systems 67: 129-145. 